\title{
Composite materials using fluorinated graphene nanoplatelets
}

\author{
A. N. Blokhin ${ }^{1, *}$, T.P. Dyachkova ${ }^{1}$, A. K. Sukhorukov ${ }^{1}$, D. E. Kobzev ${ }^{1}$, \\ E. V. Galunin ${ }^{1}$, A. V. Maksimkin ${ }^{1}$, A. S. Mostovoy ${ }^{1}$, A.P. Kharitonov ${ }^{2}$ \\ ${ }^{1}$ Tambov State Technical University, Tambov, Russia \\ ${ }^{2}$ Branch of the Talrose Institute for Energy Problems of Chemical Physics, \\ Russian Academy of Sciences, Chernogolovka, Moscow Region, Russia \\ *cha-cha@rambler.ru
}

PACS 82.35.NP

DOI 10.17586/2220-8054-2018-9-1-102-105

The changes occurring on the surface of graphene nanoplatelets (GNPs) during treatment with gaseous fluorine are shown. According to Raman and IR spectroscopy, C-F covalent bonds are formed. As the fluorination temperature increases, the destructive changes in the GNPs become more noticeable, as evidenced by the results of X-ray diffraction analysis and the specific surface area of the samples. The presence of fluorine-containing functional groups contributes to better dispersion of the GNPs in the epoxy matrix and to an increase in their strengthening effect. The epoxy composite containing $0.1 \mathrm{wt} \%$ of the GNPs treated with fluorine at $450{ }^{\circ} \mathrm{C}$ presents the maximum strength characteristics: in comparison with the unmodified material, the tensile stress increases by more than 2 times, the tensile modulus - by $20 \%$, the breaking stress at bending - by $80 \%$, and the modulus of elasticity at bending - by $60 \%$.

Keywords: grapheme nanoplatelets, fluorination, epoxycomposites, strength properties, reinforcement.

Received: 19 June 2017

\section{Introduction}

Graphene nanoplatelets (GNP) are layered structures based on graphene planes and, due to their properties, they can act as ideal filler for polymer composites.

In particular, in [1], it was shown that when injected into epoxy resins at a concentration of $5 \mathrm{wt} \%$, GNPs promote an increase in the electrical conductivity by an order of magnitude and exhibition of pronounced hydrophobic properties; however, due to poor interaction with the matrix, they practically do not affect the mechanical parameters.

To improve the GNP interaction, various functionalization methods can be used [2]. By analogy with carbon nanotubes [3], it should be expected that the fluorination of GNPs must lead to an increase in their reinforcing effect when employed as a component of epoxy composites.

The purpose of the present research was to study 1) the effect of the conditions of direct fluorination of GNPs on the chemical composition of their surface, 2) the integrity of graphene layers, 3) and the effectiveness of their application for strengthening epoxy binders.

\section{Experimental}

The GNPs (produced at NanoTechCenter Ltd., Tambov, Russia) with an average thickness of individual structural units of about $5 \mathrm{~nm}$ was used herein. They were fluorinated in a steel reactor at a pressure of gaseous fluorine of $1 \mathrm{~atm}$ at temperatures of $350-450{ }^{\circ} \mathrm{C}$. For the production of composites, a BFE-170 Bisphenol-F epoxy resin (Chang Chun Plastics Co., LTD, China) and an L-19 polyaminoamide hardener (CHIMEX Limited, Russia) were used.

Infrared (IR) spectra of the samples of the initial and fluorinated GNPs were recorded on a Nicolet 380 spectrometer. Roentgen photoelectron spectra were recorded on a ULVAC-PHI Versa Probe I device. Monochromatic Alk $\alpha$-radiation $(1486.6 \mathrm{eV})$ with a power of $50 \mathrm{~W}$ was used to excite photoemission. The powders were pressed into the In plate with a thin continuous layer. The diameter of the analysis area was $200 \mu \mathrm{m}$. X-ray diffraction patterns of the initial and fluorinated GNP were recorded on a Difrey-401 instrument with Bragg-Brentano focusing method. The specific surface area was measured by nitrogen adsorption at $77 \mathrm{~K}$ using the multipoint BET method on a Nova 1200e Quantachrome instrument. TEM images are obtained with a JOEL JEM 2000 FX microscope.

Effective sizes of GNP agglomerates in epoxy dispersions were determined by the dynamic light scattering method using a Nicomp 380 ZLS instrument. 
The mechanical properties of the epoxy-based nanocomposites were tested on a Testometric M350-5AT universal test machine. Surface images of the composites were obtained on a JEOL JSM-6610LV scanning electron microscope.

\section{Results and discussion}

As seen from the IR spectra of the fluorinated GNPs, the intensity of the absorption bands due to the presence of hydroxyl groups and/or adsorbed moisture (about $3400 \mathrm{~cm}^{-1}$ ) and carboxyl groups (about $1630 \mathrm{~cm}^{-1}$ ) decreases, indicating the removal of oxide formations present on the surface of the initial GNPs. The presence of a band at $1200 \mathrm{~cm}^{-1}$ indicates the formation of covalent bonds between the fluorine and carbon atoms.

The typical panoramic and C1s XPS spectra of the fluorinated GNP are shown in Fig. 1. The expanded spectra of photoelectronic lines have a shape characteristic of graphene-like structures: an asymmetric narrow peak at $284.4 \mathrm{eV}$, and a $\pi$ satellite at $291.0 \mathrm{eV}$. Low-intensity peaks from the oxygen bonds at $286.6 \mathrm{eV}(3.0 \%)$ and $288.5 \mathrm{eV}(1.5 \%)$ (single and double, respectively) and sulfur bond at 229.2 and $162.3 \mathrm{eV}$ can also be observed. This is due to the presence of oxygen- and sulfur-containing functional groups formed during the production of the initial GNPs.

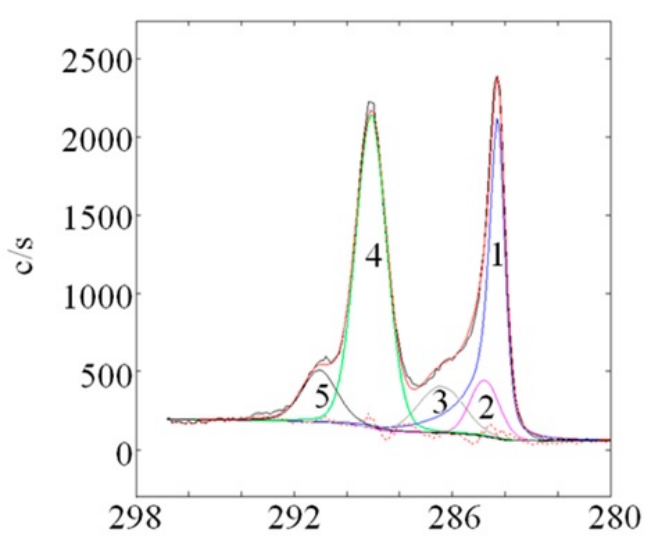

FIG. 1. Expanded XPS spectra for carbon photoelectronic lines of the GNPs subjected to direct fluorination at $350{ }^{\circ} \mathrm{C}$

In the course of the fluorination, the total amount of oxygen and sulfur in the materials decreases down to the complete removal of the sulfur-containing surface formations at 400 and $450{ }^{\circ} \mathrm{C}$. The peaks at $284.8-285.2$, 286.4-287.7, 289.1-289.2 and 291.1-291.3 eV are associated with the formation of C-F chemical bonds [4]. The preferred coordination of the carbon atoms is as follows: one bond with fluorine, and the remaining bonds with the carbon atoms also associated with fluorine. There is also a small fraction of the carbon atoms having two bonds with fluorine. About $30 \%$ of the carbon atoms in the fluorinated samples at 350 and $450{ }^{\circ} \mathrm{C}$ and $45 \%$ in the fluorinated sample at $400{ }^{\circ} \mathrm{C}$ were found not to change their initial chemical state.

No significant destructive changes in the structure of GNPs can be detected on the TEM image (Fig. 2) due to fluorination. The particles of amorphous carbon are probably visualized as black dots on the surface. According to the X-ray diffraction analysis data, the fluorination promotes disordering of the regular hexagonal structure of graphite planes and the appearance of amorphous halo due to the higher temperature of the nanographene treatment with fluorine. The specific surface area of the GNPs during the fluorination increases by 2.47 -fold, which may also be due to the availability of the amorphous phase on the GNP surface.

The average effective size of the agglomerates of the initial GNPs in the epoxy binder is about $1.2 \mu \mathrm{m}$. The GNP fluorination promotes their more uniform distribution in the matrix; the effective particle size of the dispersed phase does not exceed $900 \mathrm{~nm}$.

The changes occurring with the surface of the GNPs during the fluorination contribute to an increase in their effectiveness as a strengthening additive to the epoxy composites (Table 1).

In comparison with the initial matrix, the greatest effect is achieved when using the GNPs fluorinated (f-GNPs) at $450{ }^{\circ} \mathrm{C}(0.1 \mathrm{wt} \%)$ : the tensile stress $\left(G_{\text {tensile }}\right)$ increases by more than 2 times, the tensile modulus $\left(E_{\text {tensile }}\right)-$ by $20 \%$, the breaking stress at bending $\left(G_{\text {bending }}\right)$ - by $80 \%$, and the modulus of elasticity at bending $\left(E_{\text {bending }}\right)$ - by $60 \%$. The obtained increase in the strength is superior to that reported in the published data in the case of using the initial and functionalized graphene structures [5-7]. 


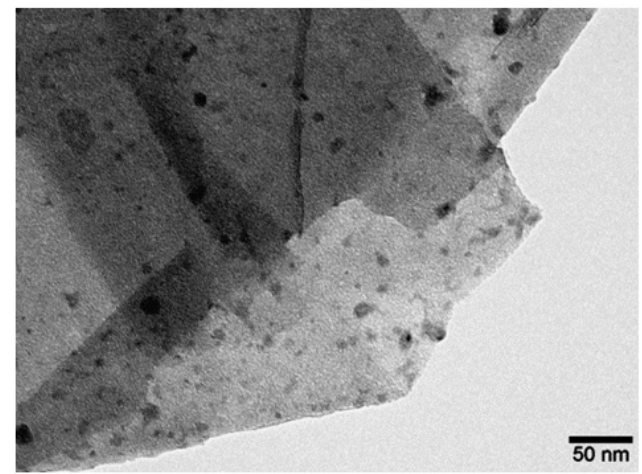

FIG. 2. TEM image of the fluorinated GNPs

TABLE 1. Results of strength tests of the BFE-170 Bisphenol-F resin-based composites

\begin{tabular}{|c|c|c|c|c|c|}
\hline Filler and mass fraction thereof & $G_{\text {tensile }}, \mathrm{MPa}$ & $E_{\text {tensile }}, \mathrm{MPa}$ & $\varepsilon, \%$ & $G_{\text {bending }}, \mathrm{MPa}$ & $E_{\text {bending }}, \mathrm{MPa}$ \\
\hline- & $38 \pm 1.8$ & $2204 \pm 88$ & $2 \pm 0.10$ & $107 \pm 5.5$ & $1683 \pm 68$ \\
\hline Initial GNPs, $0.01 \%$ & $56 \pm 2.8$ & $2260 \pm 91$ & $2.5 \pm 0.10$ & $124 \pm 6.0$ & $1850 \pm 74$ \\
\hline Initial GNPs, 0.1\% & $62 \pm 3.1$ & $2480 \pm 99$ & $3.5 \pm 0.15$ & $131 \pm 6.2$ & $2189 \pm 88$ \\
\hline Initial GNPs, $0.5 \%$ & $46 \pm 2.3$ & $2678 \pm 105$ & $2.5 \pm 0.10$ & $120 \pm 6.0$ & $2581 \pm 100$ \\
\hline f-GNPs $350{ }^{\circ} \mathrm{C}, 0.01 \%$ & $65 \pm 3.2$ & $2281 \pm 91$ & $4.2 \pm 0.17$ & $136 \pm 6.5$ & $2010 \pm 80$ \\
\hline f-GNPs $350{ }^{\circ} \mathrm{C}, 0.1 \%$ & $71 \pm 3.5$ & $2305 \pm 92$ & $5 \pm 0.20$ & $170 \pm 8.0$ & $2639 \pm 105$ \\
\hline f-GNPs $350{ }^{\circ} \mathrm{C}, 0.5 \%$ & $58 \pm 2.8$ & $2937 \pm 115$ & $2.7 \pm 0.11$ & $124 \pm 6.0$ & $2660 \pm 105$ \\
\hline f-GNPs $340{ }^{\circ} \mathrm{C}, 0.01 \%$ & $69 \pm 3.4$ & $2384 \pm 95$ & $3.5 \pm 0.15$ & $142 \pm 6.9$ & $2210 \pm 88$ \\
\hline f-GNPs $450{ }^{\circ} \mathrm{C}, 0.1 \%$ & $84 \pm 3.8$ & $2592 \pm 104$ & $5 \pm 0.20$ & $192 \pm 7.5$ & $2699 \pm 107$ \\
\hline f-GNPs $450{ }^{\circ} \mathrm{C}, 0.5 \%$ & $63 \pm 3.1$ & $2791 \pm 110$ & $3.5 \pm 0.15$ & $146 \pm 7.1$ & $2766 \pm 110$ \\
\hline
\end{tabular}

The rupture surfaces of all the composites (Fig. 3) have a structure similar to each other, independent of the presence or absence of the initial and fluorinated GNPs. The GNPs were not detected on the rupture surface of the filled nanocomposites, which can be explained by their fairly good adhesion to the epoxy matrix.

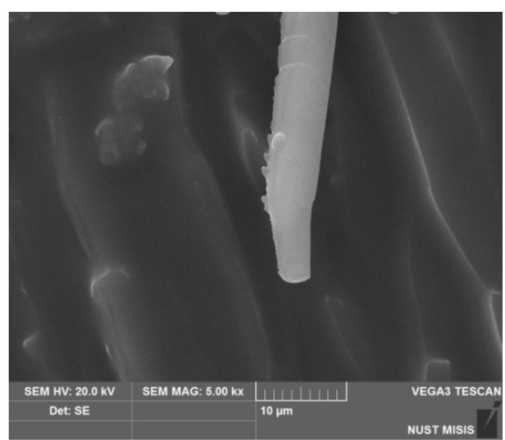

(a)

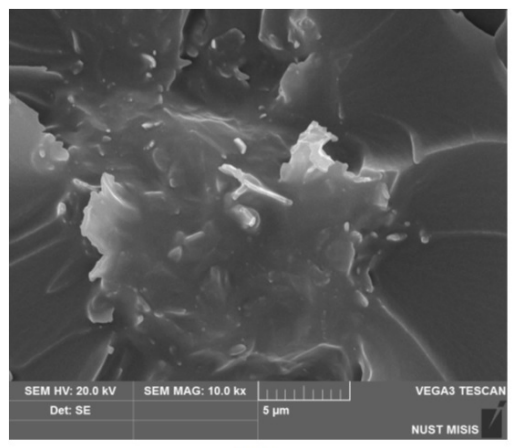

(b)

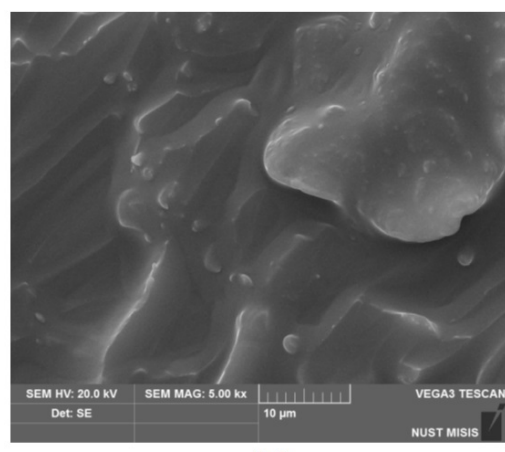

(c)

FIG. 3. SEM images of the epoxy matrix surfaces: (a) unmodified, and modified with (b) the initial and fluorinated (c) GNPs 


\section{Conclusion}

GNPs are an effective filler of epoxy matrices that contribute to the improvement of their physical and mechanical properties. GNP treatment with gaseous fluorine results in the formation of fluorine-containing surface functional groups that enhance interaction with the epoxy matrix and contribute to more uniform dispersion therein.

However, when choosing a fluorination mode, it is necessary to take into account destructive changes taking place in the GNPs. Due to the fluorination, a higher strengthening effect, surpassing the literature data, has been achieved.

\section{Acknowledgements}

The research has been supported by the Russian Science Foundation grant 15-13-10038.

\section{References}

[1] Prilongo S.G., Moriche R., Jiménez-Suárez A., Sánchez M., Ureña A. Advantages and dis-advantages of the addition of grapheme nanoplatelets to epoxy resins. Eur. Polymer J., 2014, 61, P. 206-214.

[2] Punetha V.D., Rana S., Yoo H.J., Chaurasia A., McLeskey J.T., Ramasamy M.S., Sahoo N.G., Cho J.W. Functionalization of carbon nanomaterials for advanced polymer nanocompo-sites: A comparison study between CNT and graphene. Progr. Polymer Sci., 2017, 67, P. $1-47$.

[3] Kharitonov A.P., Simbirtseva G.V., Tkachev A.G., Blohin A.N., Dyachkova T.P., Maksim-kin A.A., Chukov D.I. Reinforcement of epoxy resin composites with fluorinated carbon nano-tubes. Comp. Sci. Technol., 2015, 107, P. 162-168.

[4] Beamson G., Briggs D. High Resolution XPS of Organic Polymers. J. Chem. Educ., 1993, 70(1), P. A25.

[5] Hadden C.M., Klimek-McDonald D.R., Pineda E.J., King J.A., Reichanadter A.M., Miskioglu I., Gowtham S., Odegard G.M. Mechanical Properties of Graphene Nanoplatelet, Carbon Fiber, Epoxy Hybrid Composites: Multiscale Modeling and Experiments. Carbon, 2015, 95, P. 100-112.

[6] Ming-Yuan Shen, Tung-Yu Chang,Tsung-Han Hsieh,Yi-Luen Li,Chin-Lung Ciang, Hsiharng Yang, Ming-Chuen Yip. Mechanical Properties and Tensile Fatigue of Graphene Nanoplatelets Reinforced Polymer Nanocomposites. J. Nanomater., 2013. URL: http://downloads.hindawi.com/journals/jnm/2013/565401.pdf.

[7] Minoo Naebe, Jing Wang, Abbas Amini, Hamid Khayyam, Nishar Hameed, Lu Hua Li, Ying Chen, Bronwyn Fox. Mechanical Property and Structure of Covalent Functionalised Graphene, Epoxy Nanocomposites. Sci. Reports, 2014. URL: http: //www. nature.com/articles/srep04375.pdf. 